\title{
Research on Dynamic Warning of Miners' safety Behavior Ability in Complex Environment
}

\author{
Zhu Yanna ${ }^{1, \text { a, }{ }^{*}, \text { He Gang }}{ }^{1}$, Qiao Gongtong ${ }^{2}$ and Zhang Guisheng ${ }^{2}$ \\ ${ }^{1}$ Economics and Management College, Anhui University of Science and Technology, \\ Huainan, Anhui 232001, China \\ ${ }^{2}$ Electrical and Information Engineering College, Anhui University of Science and Technology, \\ Huainan, Anhui 232001,China \\ a571436747@qq.com
}

\begin{abstract}
Keywords: Coal mine; Complex environment; Safe behavior ability; Structural equation model; Dynamic early warning
\end{abstract}

\begin{abstract}
Many factors that affect the safety behavior of miners, it mainly focus on four major aspects of the accident caused by personal behavior factors, operating environment, equipment and facilities, economic benefits and so on. The principal component analysis method and factor analysis method are used to extract the appropriate sub-index, and use structural equation model to calculate the threshold of total index and sub-index. The most important environmental indicators that affect the safety behavior of miners, which including gas concentration, mine temperature, humidity, noise $\mathrm{dB}$, water quality indicators, dust concentration and so on. According to the above, the AT89C51 main controller is carried on the classification processing and the logic judgment to each kind of sensor output corresponding signal, and controls the LCD and the buzzer to carry out the dynamic display and the alarm. This research has a strong practical application value.
\end{abstract}

\section{Introduction}

Coal mine as one of the high risk industries, with the gradually increase of mining depth and breadth, coal mine accident level is becoming increasingly fierce. According to the State Administration of production safety supervision, From December 14 to December 27, 2015, three major accidents occurred, fifty-four people death and whereabouts unknown. Gas explosion accident caused nineteen people were missing in Hegang City, Heilongjiang Province. Because of coal mine fire occurs, which resulting in seventeen people deaths in Liaoning Province. Because of the gypsum mine collapse caused one people were killed and seventeen people missing in Pingyi City, Shandong Province. There is no doubt that the causes of these accidents can not be determined solely from a single level. Lax oversight of local government, driven by the enterprise economic interests, miners' low safety awareness, lack of safety skills, and poor working environment in a certain extent lead to accidents occurred. Therefore, it is an urgent problem for the government and enterprises to effectively identify and monitor the factors that influence the coal mines' safety behavior in complex environment.

At present, many scholars at home and abroad have made some achievements in the management of miners' safety behavior and the environment forecasting. Liu Wensheng and ZHU Ming studied on the prediction of coal mine safety by using system dynamics. Uen J F, Chien M S, Yen Y F studied the relationship between human's physiological behavior, attitude and performance, and establishes a safe behavior model based on different standards. Zhao Daiying used the safety indexes as an early warning index, using the two exponential smoothing methods to predict the early warning index, and set the threshold and level of early warning index model. Cao Qingren constructed the model of "perception-ability-behavior" based on coal miners' unsafe behavior, and summarized the factors that cause the unsafe behavior. Chen Hong analyzed and constructed the coal enterprise safety behavior choice of the force system.

To sum up, the research is mainly focused on the summary of the general concept generalization, 
ignoring the complex relationship among the factors of the system. The author uses structural equation model to analyze the safety behavior of miners in the complex environment. The AT89C51 main controller is used to classify and deal with the corresponding signals of various kinds of sensors, and to control the dynamic display and alarm of LCD and buzzer, which has strong practical application value.

\section{Constructing the Structural Equation Model of Miners' safety Behavior}

Along with the development of the early warning theory, the research of security early warning has experienced several stages, as shown in Fig. 1.

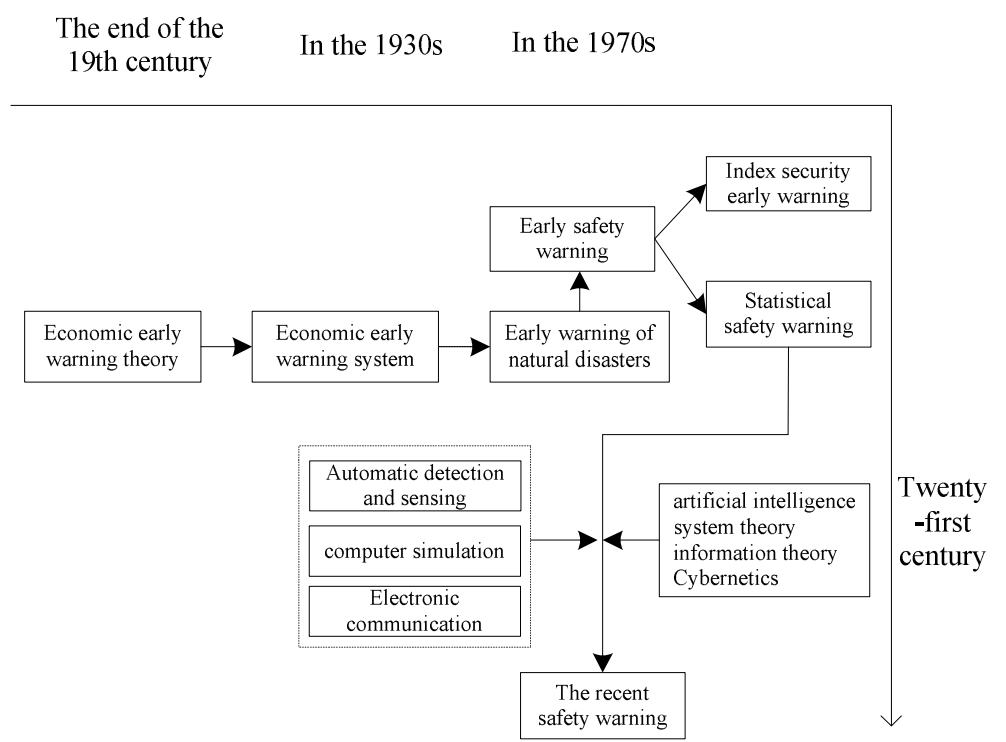

Fig. 1 The evolution and development of security early warning.

According to the security early warning evolution route, the recent early warning mechanism is mainly reflected in the security aspect. If we can find the effective early warning point of the complex coal mine environment, and to control and prevent this, it can greatly reduce the incidence of mine accidents.

The coal mining enterprises are complex, the influencing factors are involved in a wide range, if we can scientifically select the coal miners' safety impact indicators, and it will have a direct impact on the establishment of the early warning mechanism. So it is very important to design a scientific and reasonable index system on coal miners. Based on the previous research results, we use the SPSS software to analyze the factors by principal component analysis and factor analysis. Arrange and conclude that the factors affecting coal miners' safety behavior is coal mine environment level, enterprise level and employee level. Each of these factors also covers the molecular factors. Among them, the personal factors $(\xi 1)$ include: X1 post competency, X2 emergency response ability, X3 risk identification ability, X4 work experience. The mine environment $(\xi 2)$ include: Y1 dust concentration, Y2 gas concentration, Y3 temperature and humidity, Y4 noise $\mathrm{db}$. The enterprise level ( $\xi 3$ ) include: Z1 coal industry profits, Z2 the interests of local governments, Z3 owners' interests, Z4 beyond the production. The influencing factors provide the basis for the application of structural equation to construct the safety warning model and variable selection.

\section{The Empirical Analysis}

Based on the previous research results, some coal mines in Huainan, Anhui Province was selected for the relevant research. We use random sampling to extract tester. On the spot, 200 questionnaires were distributed, 190 questionnaires were recovered, the effective number of questionnaires was 186, the cumulative recovery rate was $95 \%$, and the effective recovery rate was $93 \%$. We use SPSS and 
AMOS mathematical software to calculate the reliability and validity of the data. The correlation coefficient indicates the reliability; the factor load factor of the index represents the validity.

The Test of Reliability and Validity. The results of reliability and validity are shown in Table 1 . The factor load, the reliability coefficient and the combined reliability value of each component were obtained, and the combined reliability value was more than 0.7 , which showed that the reliability and validity were acceptable.

Table 1 . The reliability and validity of the questionnaire.

\begin{tabular}{|c|c|c|c|c|}
\hline $\begin{array}{c}\text { Measurement } \\
\text { index }\end{array}$ & $\begin{array}{c}\text { Regression } \\
\text { coefficient }\end{array}$ & $\begin{array}{c}\text { Reliability } \\
\text { coefficient }\end{array}$ & $\begin{array}{c}\text { Measurement } \\
\text { error }\end{array}$ & $\begin{array}{c}\text { Combined } \\
\text { reliability }\end{array}$ \\
\hline X1 & 0.813 & 0.661 & 0.339 & \\
X2 & 0.840 & 0.706 & 0.294 & \\
X3 & 0.817 & 0.667 & 0.333 & \\
X4 & 0.690 & 0.476 & 0.524 & 0.870 \\
\hline Y1 & 0.712 & 0.507 & 0.493 & \\
Y2 & 0.716 & 0.513 & 0.487 & \\
Y3 & 0.751 & 0.564 & 0.436 & \\
Y4 & 0.780 & 0.608 & 0.392 & \\
Z1 & 0.752 & 0.566 & 0.434 & \\
Z2 & 0.781 & 0.610 & 0.390 & \\
Z3 & 0.870 & 0.757 & 0.243 & \\
Z4 & 0.827 & 0.684 & 0.316 & 0.883 \\
\hline
\end{tabular}

The Initial Model Validation. The regression coefficients were estimated by the maximum likelihood method with AMOS software, and the data were calculated according to the theoretical model of the structural equation. The results are as follows in Table 2.

Table 2. The initial verification of structural equation model.

\begin{tabular}{|cclllll|}
\hline & & & Estimate & S.E. & C.R. & P \\
\hline$\xi 1$ & $<---$ & Miners' behavior & 1.000 & & & \\
$\xi 2$ & $<---$ & Miners' behavior & 0.724 & 0.087 & 8.320 & $* * *$ \\
$\xi 3$ & $<---$ & Miners' behavior & 0.920 & 0.106 & 8.651 & $* * *$ \\
\hline X1 & $<---$ & $\xi 1$ & 1.000 & & & \\
X2 & $<---$ & $\xi 1$ & 0.922 & 0.070 & 13.185 & $* * *$ \\
X3 & $<---$ & $\xi 1$ & 0.854 & 0.067 & 12.743 & $* * *$ \\
X4 & $<---$ & $\xi 1$ & 0.761 & 0.074 & 10.293 & $* * *$ \\
\hline Y1 & $<---$ & $\xi 2$ & 1.000 & & & \\
Y2 & $<---$ & $\xi 2$ & 1.013 & 0.110 & 9.248 & $* * *$ \\
Y3 & $<---$ & $\xi 2$ & 1.064 & 0.110 & 9.665 & $* * *$ \\
Y4 & $<---$ & $\xi 2$ & 1.079 & 0.108 & 9.995 & $* * *$ \\
\hline Z1 & $<---$ & $\xi 3$ & 1.000 & & & \\
Z2 & $<---$ & $\xi 3$ & 1.030 & 0.093 & 11.128 & $* * *$ \\
Z3 & $<---$ & $\xi 3$ & 1.113 & 0.089 & 12.484 & $* * *$ \\
Z4 & $<---$ & $\xi 3$ & 0.987 & 0.083 & 11.847 & $* * *$ \\
\hline
\end{tabular}

In Table 2, the residual variable estimates of 3 potential variables and 12 error variables are positive and reach significant level. The estimated parameters have no negative error variables and 
the standard error values are very small, which indicates that the model's basic fitness is good.

The Model Operation Results. Since the initial model fitness can be successfully identified, there is no negative variation of the error, which indicates that the model is not a problem. The result of the model as is shown in Fig. 2.

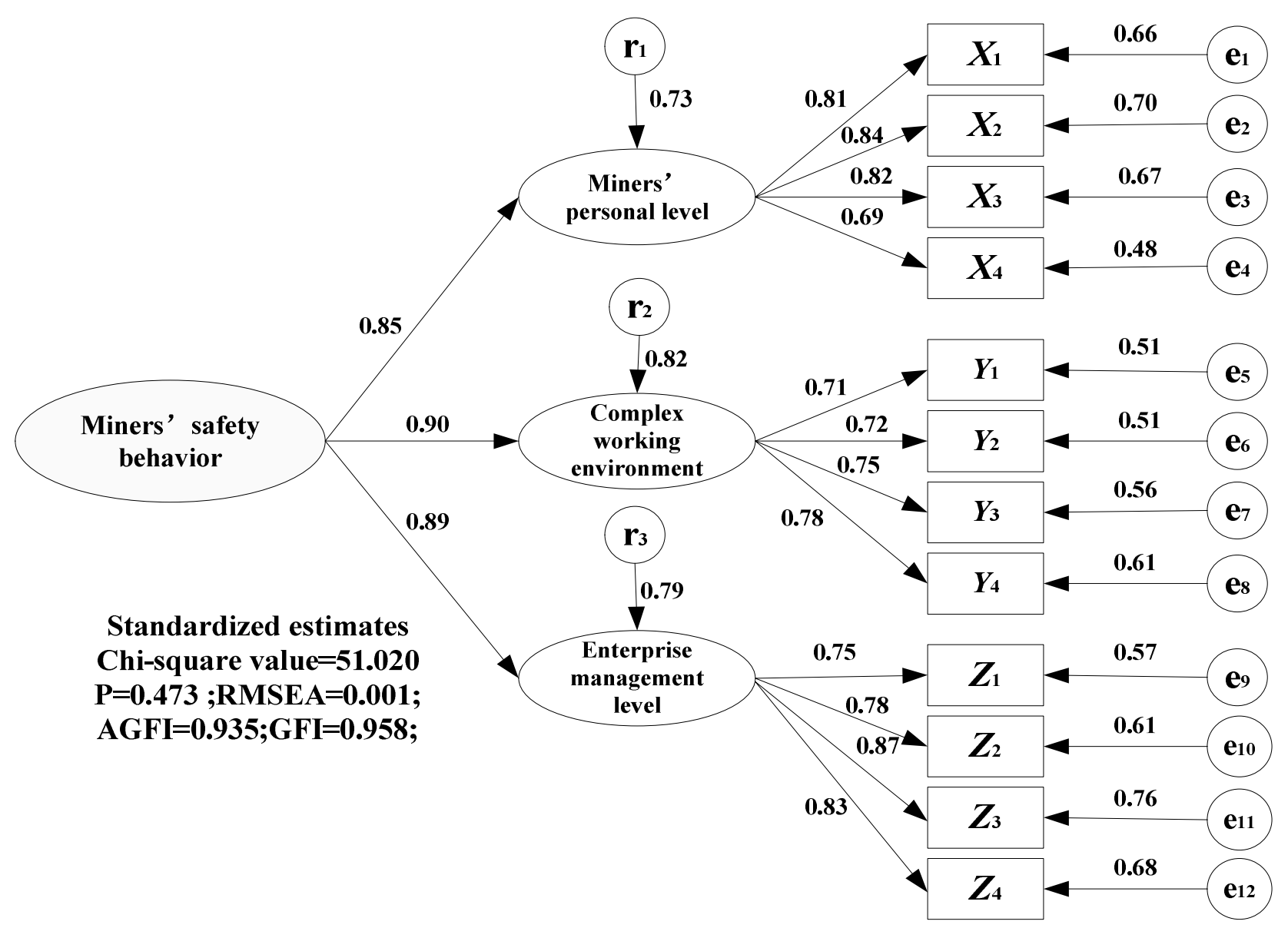

Fig. 2 Standardized estimate model.

Fig. 2 is the standardized value estimation model. From the graph can be directly observed that the three factor loadings are $0.85,0.90$ and 0.89 , which are greater than 0.7 ; the reliability index are 0.73 , 0.82 and 0.79 , it is also greater than 0.7 . This means that the reliability and validity of the model are acceptable.

According to the analysis of Fig. 2, we can know that the coal miners' personal factors, complex operating environment and enterprise management level directly affect the miners' safety behavior. The path coefficients are $0.85,0.90$ and 0.89 , respectively, and the bigger the numerical values show, the greater the influence degree is.

\section{The Construction of Early Warning Platform}

According to the analysis results, we concluded that in the three levels of the miners' safety behavior, complex operation environment and enterprise management, the influence coefficient of coal miners' complicated working environment was 0.90 , higher than the two other factors influence. Therefore, we mainly focus on miners' operating environment indicators that the biggest impact factor on safety behavior of coal miners to establish a dynamic early warning platform, warning platform as shown in Fig. 3. 


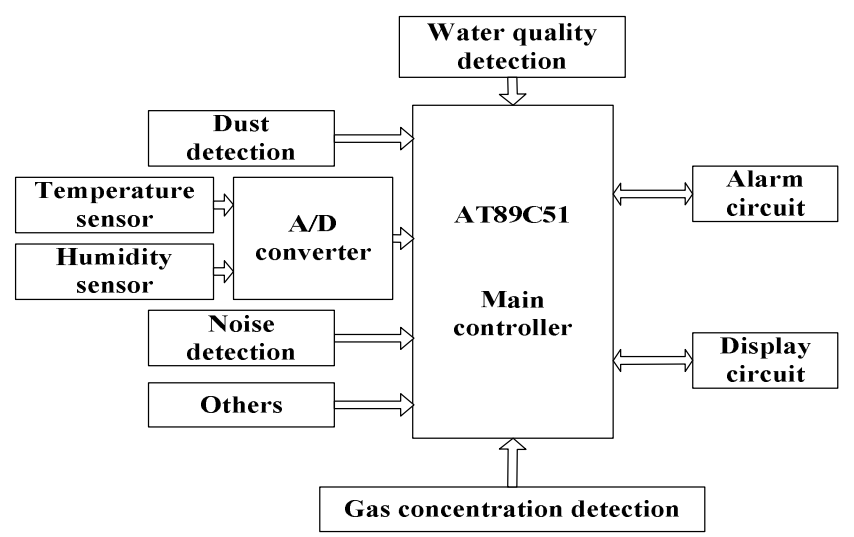

Fig. 3 Dynamic early warning platform.

The overall block diagram of the warning system is shown in Fig. 3, which is divided into four modules: the main controller, the environment detection, the alarm circuit and the display circuit. Among them, the main controller using AT89C51 micro-controller chip, its control ability is strong, the precision is high, can be a good control of environmental detection and early warning.

Environmental detection module includes water quality detection, dust detection, temperature and humidity detection, noise detection and gas concentration detection, etc. The temperature and humidity sensors are mainly used to detect the temperature and humidity in the environment of the underground workers. The sensor will detect temperature and humidity treated transformed into electrical signals, and then transported to the A / D converter, which is converted to digital signal. At last, the digital signal is transmitted to the single chip microcomputer master controller. After signal processing and logic judgment, the control command is sent out to control the display and alarm. When the temperature exceeds $40^{\circ} \mathrm{C}$, the relative humidity is more than $80 \%$ or less than $3 \%$, the device performs automatic alarm. Among them, the alarm circuit uses buzzer alarm; it can choose different alarm music according to different testing material, so as to distinguish. The circuit adopts LCD display two lines, the first line shows the name of the substance to be detected, and the second line shows the value of the detected, it is easy to read.

\section{Conclusions}

We use structural equation model to explain the influence factors and their complex relationship of the coal miners' safety behavior. The intelligent early warning system of mine operation environment based on the AT89C51 MCU control chip was established. Through the analysis of the case of coal mine, the conclusion is drawn:

1. Through the structural equation model, it can be more intuitive and quantitative to analyze the correlation coefficient between the evaluation indexes and the way of influence. The influence of different factors on miner's safety behavior is different, and the influence coefficient of complex working environment on coal miners' safety behavior is the biggest, which is 0.90 .

2. In the complex coal mine environment, the dynamic warning for the safety behavior of the miners needs a long time. Coal mining enterprises and similar high risk industries should seize the key factors that affect the safety behavior of employees, and start from the biggest impact indicator. From one side to the case, perhaps the ability to enhance the safety behavior of employees has little effect. But from the perspective of capital investment, the overall increase in the need of greater human, material and financial resources, and so on. Therefore, the most important factor is the primary choice for enterprises.

3. According to the analysis results of the structural equation model, the dynamic early warning platform for the complex working environment is built. The platform is used to control the display and early warning, and it can reflect the actual effect of its contribution to the system.

In short, if we can establish an effective dynamic early warning platform, and be able to implement a reasonable safety input structure, which will greatly reduce the incidence of coal mine accidents. This is our next step of the research direction. 


\section{Acknowledgement}

This work is supported by the following programs: the National Natural Science Foundation of China with the title "Evaluation of Coal Miners' Safety Behavioral Ability and Its Dynamic Pre-warning under the Interaction between Individuals and Environment" (NO.51574010); Research Project of the key base of Humanities and Social Sciences in Anhui Province (NO. SK2014A041).

\section{References}

[1] The State Administration of Production Safety Supervision and Management. 2016. State Administration of Safety Supervision issued in November the situation and safety of the situation analysis. [Online]. Available: http://www.chinasafety.gov.cn/newpage/Contents/Channel_4178/201 5/1218/262156/content_262156.htm.

[2] The Statistics Division of Safety Supervision and Administration. 2015. The situation of Safety production in December $27^{\text {th }}-$ December 21. [Online]. Available:http://news.youth.cn/jsxw/201512 /t20151230.htm.

[3] The central broadcasting network. 2015. Gypsum mine collapse accident in Pingyi city Shandong province.[Online].Available:http://news.youth.cn/jsxw/201512/t20151230_747940.htm.

[4] W.S. Liu, M. Zhu, 2012. The study of coalmine safety prediction based on System dynamics. 1st International Conference on Intelligent System and Applied Material (GSAM 2012) Jan 13-15, 2012. Taiyuan, PEOPLESR CHINA, 1045-1049.

[5] J. F. Uen, M. S. Chien, Y. F. Yen, The mediating effects of psychological contracts on the relationship between human resource systems and role behaviors: A multilevel analysis. J. Bus. Psych. 24(2) (2009) 215-223.

[6] D. Y. Zhao, X. Q. He, T. H. Jiang, Research on forewarning model for work safety of coal mine in China, J. Safety Sci. Technol. (1) (2014) 81-86.

[7] Q. R. Cao, S. Li, X. F. Song, Study on the KAA Unsafe Behavior Model of Coal Mine Employees, China Safety Sci. J. (12) (2007) 19-25.

[8] H. Chen, H. Qi, Driver analysis of behavior of coal enterprise security, China Mining Magazine, 18(9) (2009) 79-82. 